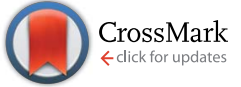

Cite this: RSC Adv., 2017, 7, 14618

Received 5th January 2017 Accepted 26th February 2017

DOI: 10.1039/c7ra00160f

rsc.li/rsc-advances

\title{
Different-sized black phosphorus nanosheets with good cytocompatibility and high photothermal performance $\uparrow$
}

\author{
Haidi Fu, ${ }^{\text {ab }}$ Zhibin Li, ${ }^{\text {bc }}$ Hanhan Xie, ${ }^{\text {b }}$ Zhengbo Sun, ${ }^{\text {b }}$ Beike Wang, ${ }^{\text {a Hao Huang, }}{ }^{\text {b }}$ \\ Guangli Han, ${ }^{* a}$ Huaiyu Wang, ${ }^{b}$ Paul K. Chu ${ }^{c}$ and Xue-Feng Yu ${ }^{b}$
}

\begin{abstract}
As a new kind of two-dimensional (2D) material, black phosphorus (BP) has attracted explosive interest in biomedical applications. It is well-recognized that the biomedical performances of nanoparticles depend not only on their properties, but also on their structures and dimensions. Here in this study, we successfully prepared three kinds of BP nanosheets with different sizes, then systematically investigated their cytocompatibility and photothermal effects to ablate cancer cells. It is evident from various assays that all the three BP samples have excellent cytocompatibility, and the BP nanosheets with a large size have better photothermal efficiency for cancer cell ablation. Our findings may provide a new insight into the fundamental cyto-performances of BP.
\end{abstract}

\section{Introduction}

Black phosphorus (BP), which was discovered as early as in 1914, is the most stable allotrope of phosphorus., ${ }^{\mathbf{1} 2}$ Nevertheless, BP had not attracted much attention over the past century, until it was recognized as a new member of two-dimensional (2D) materials family in 2014. ${ }^{3,4}$ BP is composed of puckered layers via weak van der Waals forces, thereby can be readily exfoliated into mono- or few-layer BP nanosheets by some physical or chemical methods. ${ }^{5-11}$ On account of the fascinating characteristics including tunable bandgap, ${ }^{12-14}$ high carrier mobility ${ }^{15}$ robust field emitter nature, ${ }^{\mathbf{1 6 - 1 8}}$ and the anisotropic photoelectronic properties, ${ }^{19,20} \mathrm{BP}$ nanosheets with atomic thickness have invoked a research boom in various applications, such as field-effect transistors, ${ }^{12,21,22}$ lithium-ion batteries, ${ }^{23,24}$ memory devices, ${ }^{25}$ diodes, ${ }^{26,27}$ phototransistors, ${ }^{4,12}$ photovoltaics, ${ }^{28-30}$ photodetectors, $^{31,32}$ humidity sensors ${ }^{10,33}$ and supercapacitors. ${ }^{34}$

Apart from the tremendous potentials mentioned above, BP is also a promising candidate for biomedical applications, ${ }^{\mathbf{8}, 35-37}$ as that phosphorus is an essential element for human health. More importantly, BP can be degraded in vivo, ${ }^{38-40}$ and the final

${ }^{a}$ State Key Laboratory Breeding Base of Basic Science of Stomatology (Hubei-MOST), Key Laboratory for Oral Biomedicine of Ministry of Education (KLOBM) \& Department of Orthodontics, School and Hospital of Stomatology, Wuhan University, 237 Luoyu Road, Wuhan 430079, P. R. China. E-mail: guanglihan@ hotmail.com

${ }^{b}$ Institute of Biomedicine and Biotechnology, Shenzhen Institutes of Advanced Technology, Chinese Academy of Sciences, Shenzhen 518055, P. R. China

${ }^{c}$ Department of Physics and Materials Science, City University of Hong Kong, Tat Chee Avenue, Kowloon, Hong Kong, P. R. China

† Electronic supplementary information (ESI) available. See DOI: 10.1039/c7ra00160f degradation product phosphorus oxides can be involved in the energy supply of organisms. Our previous studies have reported that BP quantum dots (ultrasmall BP nanosheets) and BPQDs@PLGA nanospheres had excellent near-infrared (NIR) photothermal performance of cancer therapy. ${ }^{\mathbf{8}, 36}$ There is also another study demonstrated that BPQDs possessed excellent NIR photoacoustic properties, ${ }^{37}$ which is satisfactory for the bioimaging applications.

Despite of the unprecedented achievements recently, the researches of BP in biomedical applications are still in their infancy with many unresolved but fundamental concerns. As aforementioned, there are different physical and chemical methods for BP exfoliation, and the BP nanomaterials can be prepared in different dimensions. It is well-known that the organisms such as cells are very sensitive to nanomaterials, when the size, shape and surface coating of nanomaterials can trigger different cellular responses through endocytic pathways. ${ }^{\mathbf{4 1}}$ Here in this study, we have prepared the BP nanosheets in different sizes, then systematically investigated the size effect of the BP on cytocompatibility. Additionally, the BP nanosheets in different sizes are compared for their photothermal ability of tumor cells ablation. This work may provide a new insight into the fundamental cyto-performances of BP nanosheets.

\section{Experimental}

\subsection{Materials}

The BP crystals were obtained from a commercial supplier (Smart-Elements) and stored in a dark Ar glovebox. Dimethyl sulfoxide (DMSO, 99.95\%, anhydrous) was obtained from Aladdin Reagents. The Dulbecco's Modified Eagle medium (DMEM), fetal bovine serum (FBS), phosphate buffer saline (PBS 
pH 7.4) and trypsin-EDTA were obtained from Gibco Life Technologies (AG, Switzerland). Cell-Counting Kit-8 (CCK-8), Calcein-AM/Propidium iodide (PI) Double Stain Kit, Annexin V-fluorescein isothiocyanate (Annexin V-FITC)/PI cell apoptosis detection kit were obtained from TransGen Biotech Co., Ltd. Ultrapure water $\left(18.25 \mathrm{M} \Omega \mathrm{cm}, 25^{\circ} \mathrm{C}\right)$ was used as the solvent in all the experiments. All the chemicals used in this study were analytical reagent grade and used without further purification.

\subsection{Synthesis and characterization}

2.2.1. Synthesis of different-sized BP nanosheets. The BP nanosheets were prepared by an ameliorated liquid exfoliation technique referred to our previous study. ${ }^{\mathbf{8 , 4 2}}$ In brief, the bulk BP (25 mg) was added to DMSO $(25 \mathrm{~mL})$ in a $50 \mathrm{~mL}$ sealed conical tube, and the mixture was sonicated with a sonic tip at the power of $1200 \mathrm{~W}$. The ultrasonic frequency was from 19 to 25 $\mathrm{kHz}$ and the ultrasound probe worked $2 \mathrm{~s}$ with an interval of $4 \mathrm{~s}$. The dispersion was then sonicated in an ultrasonic bath continuously for another $10 \mathrm{~h}$ at the power of $300 \mathrm{~W}$. The temperature of sample solution was kept below $277 \mathrm{~K}$ by an ice bath. We change the ultrasound time and centrifugal speed to obtain BP nanosheets in different sizes. After a $3 \mathrm{~h}$ of tipsonication exfoliation and another $10 \mathrm{~h}$ of water-bath ultrasound, the solution was centrifuged at $5000 \mathrm{rpm}$ for $20 \mathrm{~min}$ to remove the non-exfoliated bulk BP. Afterwards, the supernatant was then centrifuged at $7000 \mathrm{rpm}$ for another $20 \mathrm{~min}$ to precipitate the relatively large size BP nanosheets (designated as L-BP) from the DMSO. The supernatant was further centrifuged at $12000 \mathrm{rpm}$ for $20 \mathrm{~min}$ to separate the relatively small size BP quantum dots (designated as S-BP). The BP nanosheets in medial size (designated as M-BP) was synthesised by tipsonication for $1.5 \mathrm{~h}$ and subsequent water-bath ultrasound for $10 \mathrm{~h}$. The resultant dispersion was centrifuged for $20 \mathrm{~min}$ at $7000 \mathrm{rpm}$ and removed the precipitation, then the supernatant was centrifuged for $20 \mathrm{~min}$ at $10000 \mathrm{rpm}$ to obtain M-BP. All the BP nanosheets obtained were cleaned twice with absolute ethanol and washed with sterile water for three times to remove organic solvents completely and re-suspended in the aqueous solution.

2.2.2. Characterization. The transmission electron microscopy (TEM) images were acquired on the Tecnai G2 F20 $\mathrm{S}$-Twin transmission electron microscope at an acceleration voltage of $200 \mathrm{kV}$. The scanning electron microscope (SEM) images were obtained on the field-emission scanning electron microscope (NOVA NANOSEM430, FEI, Netherlands). The sizes of different BP samples were determined by measuring 200 particles according to the SEM or TEM images. The atomic force microscopy (AFM) images were performed on the drop-cast flakes on $\mathrm{Si} / \mathrm{SiO}_{2}$ substrates using an MFP-3D-S atomic force microscope (Asylum Research, USA) under the AC mode (tapping mode) in air. Raman scattering was performed on a Horiba Jobin-Yvon Lab Ram HR VIS high-resolution confocal Raman microscope equipped with a $633 \mathrm{~nm}$ laser as the excitation source at room temperature. The BP concentration was determined by inductively coupled plasma atomic emission spectroscopy (ICP-AES, IRIS Intrepid II XSP, thermo Electron
Corporation, USA). Ultraviolet-visible-near infrared (UV-vis-NIR) absorption spectra were recorded on a Lambda25 spectrophotometer (PerkinElmer) with QS-grade quartz cuvettes at room temperature. The optical absorbance per cell length $(A / L)$ was determined from the optical absorbance intensity at $808 \mathrm{~nm}$. The BP extinction coefficient was extracted from the slope of a plot of $A / L$ versus concentration by using Beer's law $(A / L=\alpha C)$.

2.2.3. NIR photothermal performance. The NIR photothermal effect was measured on a custom setup by employing a fiber-coupled continuous semiconductor diode laser $(808 \mathrm{~nm}$, KS-810F-8000, Kai Site Electronic Technology Co., Ltd. Shanxi, China). Particularly, $1 \mathrm{~mL}$ of the sample in a $1 \mathrm{~cm}$ path length quartz cuvette was irradiated by the laser with a power density of $1.0 \mathrm{~W} \mathrm{~cm}^{-2}$ for $10 \mathrm{~min}$. The laser spot was adjusted to cover the entire surface of the sample when an infrared thermal imaging camera (Fluke Ti27, USA) was used to monitor the temperature change.

\subsection{Cell cultures}

The LO2 cells (human normal hepatocyte), Hela cells (human cervical cancer cells) and MCF-7 cells (human breast cancer cells) obtained from ATCC (American Type Culture Collection) were cultured in a DMEM medium supplemented with $10 \%(\mathrm{v} / \mathrm{v})$ fetal bovine serum (Gibco), $100 \mathrm{U} \mathrm{mL}^{-1}$ penicillin, and $100 \mathrm{U}$ $\mathrm{mL}^{-1}$ streptomycin in a humidified atmosphere of $5 \% \mathrm{CO}_{2}$ at $37^{\circ} \mathrm{C}$.

To evaluate the cellular response of BP nanosheets, the various BP samples were suspended in DMEM and diluted to different concentrations $\left(6.3,12.5,25.0\right.$ and $\left.50.0 \mu \mathrm{g} \mathrm{mL} \mathrm{m}^{-1}\right)$ initially. The BP suspension was sonicated for $30 \mathrm{~min}$ at $100 \mathrm{~W}$ before adding to the cells.

\subsection{Cell assays}

2.4.1. Live/dead cells staining. A Live/Dead Cell Viability assay was involved in this study according to the manufacture's instruction. Particularly, the cells cultured with $25.0 \mu \mathrm{g} \mathrm{mL} \mathrm{m}^{-1}$ different BP samples for $24 \mathrm{~h}$ were co-stained with calcein AM and PI for $30 \mathrm{~min}$. Afterwards, the stained cells were rinsed twice with PBS and then examined by an Olympus IX71 motorized inverted microscope when the cells without BP treatment served as the control.

2.4.2. Assay of cell viability. CCK-8 assay was employed to determine the viability of different cells after the co-incubation with different BP nanosheets. Typically, $100 \mu \mathrm{L}$ cell suspension was seeded onto 96-well plates with a cell concentration of 5000 cells per well and incubated overnight. Subsequently, the medium was refreshed with the culture media containing different BP nanosheets in various concentration $(6.3,12.5,25.0$ and $50.0 \mu \mathrm{g} \mathrm{mL}^{-1}$ ). After 12,24 and $48 \mathrm{~h}$ of co-incubation, the cells were washed twice with PBS then $100 \mu \mathrm{L}$ CCK-8 solution (10\% in culture medium) was added onto each well and incubated for another $2 \mathrm{~h}$ at $37{ }^{\circ} \mathrm{C}$. To avoid interference from residual $\mathrm{BP}, 80 \mu \mathrm{L}$ supernatant of each well was transferred to a new 96-well plate before the final absorbance measurement. The absorbance was measured at a wavelength of $450 \mathrm{~nm}$ by using a microplate reader (Varioskan Flash 4.00.53, Finland). 
The cell viability was normalized to the control group and the following formula was used to evaluate cell viability: cell viability $(\%)=$ (mean of Abs. value of treatment group $/$ mean Abs. value of control) $\times 100 \%$. For each samples, the cell viability assay was performed in quintuplicate.

2.4.3. Assay of cell apoptosis and necrosis. To detect cell apoptosis and necrosis, Annexin V-FITC/PI apoptosis assay (TransGen Biotech) was involved. In brief, LO2 cells at a density of $1.0 \times 10^{5}$ cells per mL were seeded onto the 24 well-plate and cultured overnight. Subsequently, the cells were treated with $25.0 \mu \mathrm{g} \mathrm{mL}{ }^{-1} \mathrm{BP}$ samples for $24 \mathrm{~h}$. Finally, the cells were harvested, rinsed thrice with PBS, stained with Annexin V-FITC and PI (Annexin V-FITC labeled apoptotic cells while PI labeled necrotic cells), and analyzed by a cell Lab Quanta SC flow cytometry (Beckman coulter, USA).

\subsection{In vitro photothermal experiments}

The MCF-7 cells $\left(1.0 \times 10^{5}\right.$ cells per well $)$ were seeded onto the 24-well plates and incubated overnight. Subsequently, the cells were added with 12.5 and $25.0 \mu \mathrm{g} \mathrm{mL}{ }^{-1}$ different BP samples for another $4 \mathrm{~h}$ of incubation, and then illuminated with the $808 \mathrm{~nm}$ laser $\left(1.0 \mathrm{~W} \mathrm{~cm}^{-2}\right)$ for $15 \mathrm{~min}$. The laser spot was adjusted to fully cover the area of each well. After irradiation, the cells were incubation for another $12 \mathrm{~h}$, rinsed with PBS, and detected by a Live/Dead Cell Viability assay according to the manufacture's instructions. The cells were examined by an Olympus IX71 motorized inverted microscope when the cells without BP treatment served as the control.

\subsection{Statistical analysis}

The values were presented as mean or mean \pm standard deviation (SD). All the experiments were performed at least three times with data from a typical experiment shown. One-way analysis of variance (ANOVA) combined with the Bonferroni's post-test was utilized to determine the level of significance with $p<0.05$ considered to be significantly different.

\section{Results and discussion}

\subsection{Characterization}

The BP nanosheets with different sizes were prepared by an ameliorated liquid exfoliation technique as aforementioned. ${ }^{\mathbf{8 , 4 2}}$ The ultrasound time and centrifugal speed were tuned to obtain the BP nanosheets in different sizes, which were characterized by TEM and SEM. As shown in Fig. $1 \mathrm{a}-\mathrm{c}$ and $\mathrm{j}$, the obtained L-BP sample is in the shape of nanosheets with average size about $394 \pm 75 \mathrm{~nm}$. Fig. 1d-f and k reveal that the average size of M-BP is about $118 \pm 22 \mathrm{~nm}$, and the S-BP sample is with the average size about $4.5 \pm 0.6 \mathrm{~nm}$ (Fig. 1g-i and l). The high-resolution TEM (HRTEM) image in Fig. S1 (ESI†) shows lattice fringes of $0.25 \mathrm{~nm}$, which is ascribed to the (021) plane of the BP crystal. Following the SEM and TEM determinations, all the BP samples were characterized by AFM to get an estimation of sample thickness. As the results shown together in Fig. 1m-r, the heights of L-BP, M-BP and S-BP are in the range of 15-18 nm, 6$7 \mathrm{~nm}$ and $2-3 \mathrm{~nm}$, respectively.

\subsection{Optical properties}

The L-BP, M-BP, and S-BP nanosheets at different concentrations are determined for absorbance spectra with the results shown in Fig. 2a-c. Similar to other 2D layered materials such as graphene oxide $(\mathrm{GO})^{43}$ and $\mathrm{WS}_{2},{ }^{44,45}$ all the three kinds of $\mathrm{BP}$ nanosheets showed a broad absorption band across the UV and NIR regions. According to the Lambert-Beer law: $A / L=\alpha C$, where $A$ is the absorbance of different BP samples, $L$ is the length of the cuvette, $C$ is the concentration, and $\alpha$ is the corresponding extinction coefficient, a linear trend was observed for the dependence of $A / L$ on the concentration, and the extinction coefficient $(\alpha)$ for L-BP, M-BP, and S-BP are estimated to be $32.56,21.79,15.43 \mathrm{~L} \mathrm{~g}^{-1} \mathrm{~cm}^{-1}$, respectively. It is evident that the extinction coefficient for L-BP sample is higher than the values determined from $\mathrm{M}$-BP and S-BP. On the basis of Lambert-Beer law: $A / L=\alpha C$, the absorption intensity of $25.0 \mu \mathrm{g}$ $\mathrm{mL}^{-1} \mathrm{~L}-\mathrm{BP}, \mathrm{M}-\mathrm{BP}$ and S-BP at $808 \mathrm{~nm}$ is $0.78,0.51$ and 0.34 , respectively.

To evaluate the NIR photothermal properties of L-BP, M-BP, and $\mathrm{S}-\mathrm{BP}$, the various $\mathrm{BP}$ samples are dispersed in water at different concentrations $\left(12.5,25.0\right.$ and $\left.50.0 \mu \mathrm{g} \mathrm{mL}{ }^{-1}\right)$, then exposed to an $808 \mathrm{~nm}$ NIR laser (power density: $1.0 \mathrm{~W} \mathrm{~cm}^{-2}$ ). The solution temperature is then monitored as a function of irradiation time. As shown in Fig. $2 \mathrm{~d}$ and S2 (ESI $\dagger$ ), all the three kinds of BP samples can rapidly and efficiently convert NIR light into thermal energy, and L-BP is more efficient than M-BP and $\mathrm{S}-\mathrm{BP}$ in photothermal conservation, in accordance with its higher extinction coefficient. When the concentration of L-BP is $25.0 \mu \mathrm{g} \mathrm{mL}^{-1}$, the solution temperature can increase by $24.0^{\circ} \mathrm{C}$ after $808 \mathrm{~nm}$ laser irradiation for $10 \mathrm{~min}$ (see Fig. 2d). In contrast, the temperature increase of M-BP and S-BP solution is $21.8^{\circ} \mathrm{C}$ and $19.2^{\circ} \mathrm{C}$, respectively.

The various kinds of BP nanosheets as well as bulk BP are also characterized by Raman spectroscopy. As shown in Fig. 2e, all the samples are prominent for three Raman peaks, which can be assigned to one out-of-plane phonon mode $\left(\mathrm{A}_{\mathrm{g}}^{1}\right)$ and two in-plane modes $\left(\mathrm{B}_{2 \mathrm{~g}}\right.$ and $\mathrm{A}_{\mathrm{g}}^{2}$ ) of $\mathrm{BP}$, respectively. Compared to bulk BP, the corresponding Raman peaks from L-BP, M-BP, S-BP are blue-shifted to some extent and the Raman blue-shift of SBP is the most obvious. The Raman characterization is in line with the previous SEM and TEM observations, indicating that SBP is smaller and thinner than L-BP and M-BP.

The BP samples with different sizes are examined for the stability in DMSO and the results are shown together in Fig. S3 $(\mathrm{ESI} \dagger)$. Even when the storage time is up to 1 week, the samples retain over $90 \%$ of their initial absorbance intensity at $808 \mathrm{~nm}$. Furthermore, the L-BP, M-BP and S-BP samples are separately suspended in distilled water and cell culture media to evaluate the dispersibility and colloidal stability. Fig. $2 \mathrm{f}$ reveals that all the three kinds of BP nanosheets are well-dispersed in both water and cell-culture media, no aggregation can be found after the solutions standing at room temperature for $6 \mathrm{~h}$.

\subsection{Cytocompatibility}

It is well-recognized that nanomaterials used for biomedicine must be sufficient in biocompatibility. ${ }^{46,47}$ In this regard, the 

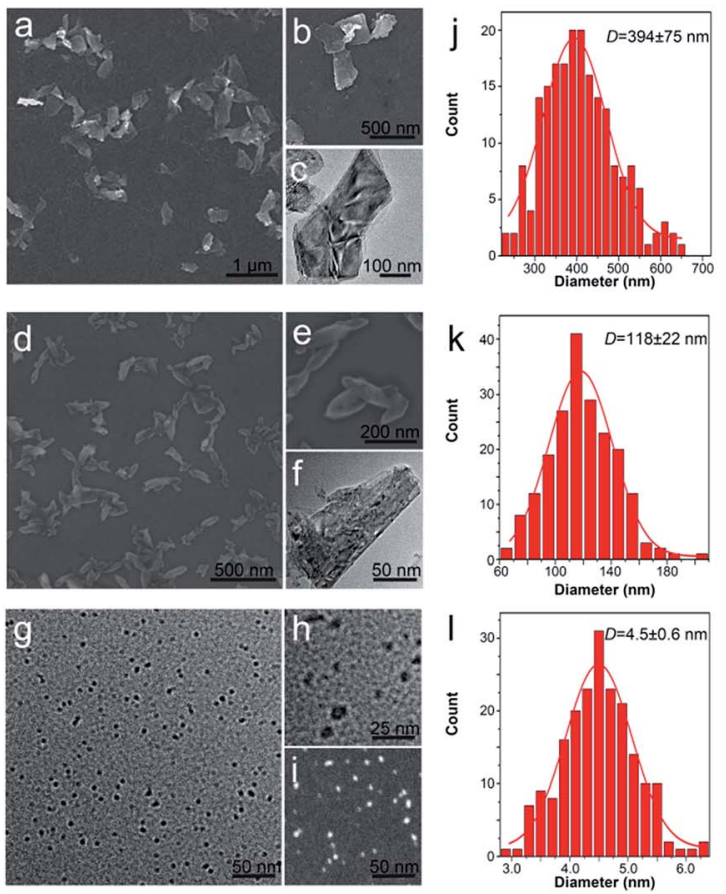
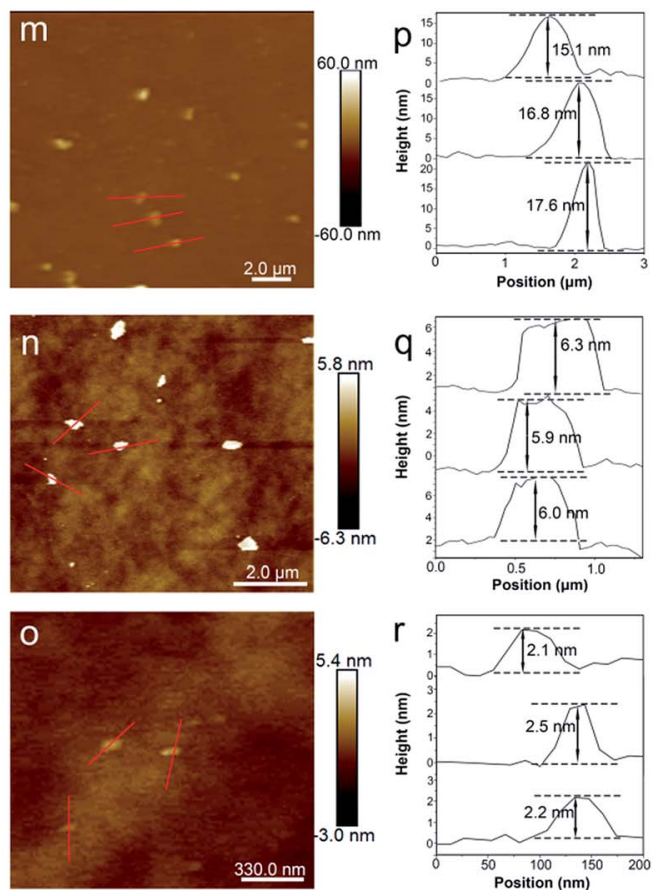

Fig. 1 Characterizations. (a and b) SEM images of L-BP; (c) TEM image of L-BP; ( $d$ and e) SEM images of M-BP; ( $f$ ) TEM image of M-BP; ( $g$ and $h$ ) TEM images of S-BP; (i) high-angle annular dark field image of S-BP. Statistical analysis of the sizes of 200 L-BP (j), M-BP (k), S-BP (l) determined by SEM or TEM. Typical AFM images and the corresponding height profiles of L-BP ( $m$ and $p$ ); $M-B P(n$ and $q$ ) and S-BP (o and $r$ ).

cytocompatibility of L-BP, M-BP, and S-BP are further evaluated by incubating them with mammalian cells. As is well known, liver is the most dominant organ for metabolism, ${ }^{48-50}$ hence
LO2, a type of human normal hepatocyte is chosen as the in vitro model in this study for the following cellular performances. Initially, the LO2 cells are incubated with different-sized BP
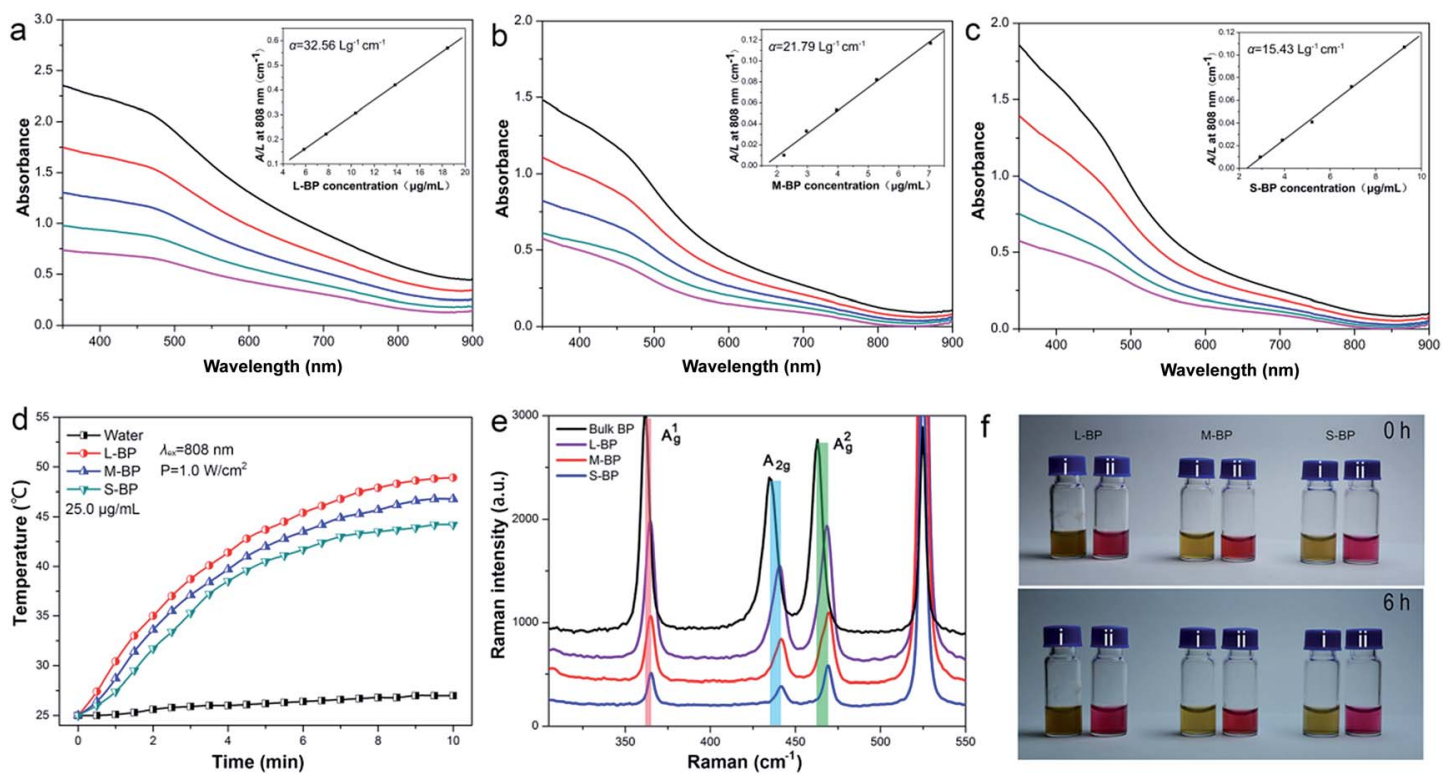

Fig. 2 Optical properties. (a-c) Absorbance spectra of L-BP (a), M-BP (b), S-BP (c) dispersed in water at different concentrations. The top curve of each graph corresponding to an initial BP concentration of $18.5,7.1,9.3 \mu \mathrm{g} \mathrm{mL}^{-1}$, respectively, determined by ICP-AES. Each subsequent curve corresponding to the BP suspension $3: 1$ diluted by distilled water. Inset: normalized absorbance intensity divided by the characteristic length of the cell $(A / L)$ at different concentrations for $\lambda=808 \mathrm{~nm}$. (d) Photothermal heating curves of distilled water and $25.0 \mu \mathrm{g} \mathrm{mL}{ }^{-1}$ of $L-B P, M-B P, S-B P$ water suspension irradiated with an $808 \mathrm{~nm}$ laser $\left(1.0 \mathrm{~W} \mathrm{~cm}^{-2}\right.$ ). (e) Raman spectra of bulk BP, L-BP, M-BP, and S-BP. (f) Photographs of L-BP, M$\mathrm{BP}$, and S-BP dispersed in water and cell culture medium for $0 \mathrm{~h}$ (upper) and $6 \mathrm{~h}$ (lower). 


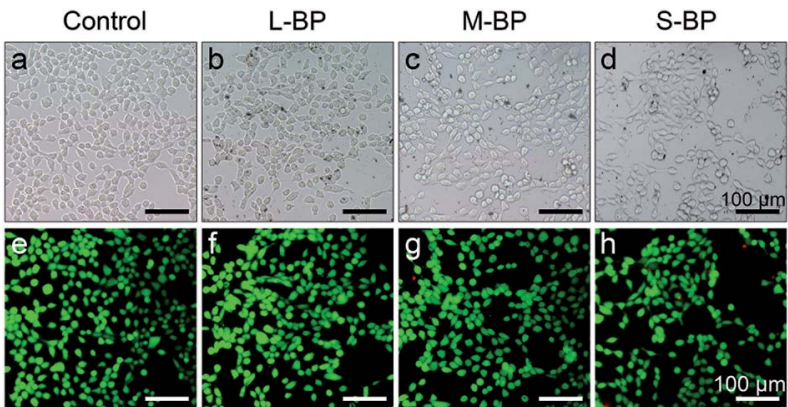

Fig. 3 Optical observation (a-d) and Live/Dead staining assay (e-h) of LO2 cells before and after the incubation with $25.0 \mu \mathrm{g} \mathrm{mL}^{-1}$ of the three BP nanosheets for $24 \mathrm{~h}$. (a and e) Control group (without the addition of BP nanosheets); ( $b$ and f) L-BP group; (c and g) M-BP group; and (d and h) S-BP group.

nanosheets $\left(25.0 \mu \mathrm{g} \mathrm{mL}^{-1}\right)$, then examine for the cell morphology by optical microscopy. As shown in Fig. 3a-d, the cells without any treatment are in normal spindle-shape, and the alteration of cell morphology is negligible after the addition of various BP samples. Noticeably, there are other dark matters in the visions of Fig. $3 \mathrm{~b}-\mathrm{d}$, which can be attributed to the aggregation of $\mathrm{BP}$ nanosheets. The $\mathrm{LO} 2$ cells of each group are further determined by a Live/Dead staining assay, where the live cells fluoresce in green (stained by calcein AM) and the dead cells fluoresce in red (stained by PI). Fig. 3e-h demonstrate that the addition of various BP nanosheets does not compromise the viability of $\mathrm{LO} 2$ cells, as vast majority of the cells are in green fluorescence. It is evident that all the three kinds of BP nanosheets are compatible to mammalian cells.
Besides the optical observation and Live/Dead staining assay, a colorimetric CCK-8 assay is also involved to evaluate the time-dependent viability (12, 24 and $48 \mathrm{~h}$ ) of LO2 cells after the incubation with L-BP, M-BP, and S-BP at different concentrations $\left(6.3,12.5,25.0\right.$ and $\left.50.0 \mu \mathrm{g} \mathrm{mL}^{-1}\right)$. As shown in Fig. $4 \mathrm{a}$, the relative cell viability of various BP groups will be little compromised when the BP concentration increases from 6.3 to $50.0 \mu \mathrm{g} \mathrm{mL} \mathrm{m}^{-1}$. Nevertheless, the cytotoxicity for all the three kinds of BP nanosheets, especially for L-BP, is non-significant. The relative cell viability of each group remains above $80 \%$, even when the BP concentration is as high as $50.0 \mu \mathrm{g} \mathrm{mL}^{-1}$ and the incubation time is up to $48 \mathrm{~h}$. Fig. $4 \mathrm{a}$ is consistent with the results shown in Fig. S4 (ESI $\dagger$ ) by involving the other 2 kinds of mammalian cells in CCK-8 assay, the low cytotoxicities of various BP samples are further corroborated. The cytocompatibility of various BP nanosheets is further determined by the flow cytometry analysis. The results in Fig. $4 \mathrm{~b}$ illustrate that the rate of cell apoptosis and necrosis is not significantly increased after the incubation of $\mathrm{LO} 2$ cells with different-sized BP nanosheets with the concentrations of $25.0 \mu \mathrm{g} \mathrm{mL}^{-1}$ for $24 \mathrm{~h}$. It is proven that all the three kinds of BP samples, especially the LBP sample, are good in cytocompatibility.

\subsection{In vitro photothermal performances}

As aforementioned, the three BP nanosheets prepared in this study are efficient in photothermal conversion (Fig. 2d). In this respect, the L-BP, M-BP and S-BP samples are further investigated for their photothermal effects for cancer cells ablation. In particular, human breast cancer cells MCF-7 are incubated with the BP samples for $4 \mathrm{~h}$, then illuminated with an NIR laser
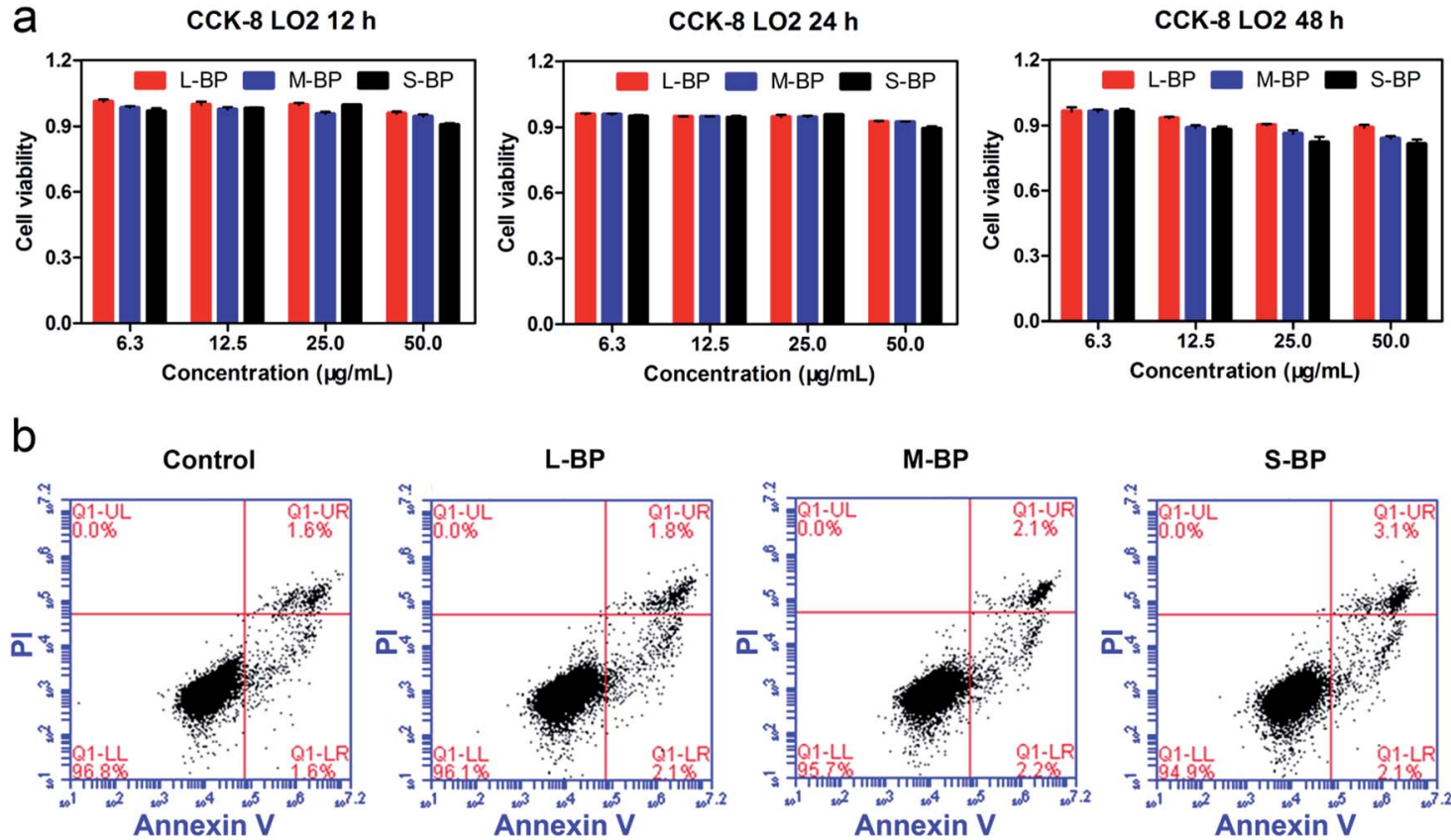

Fig. 4 CCK-8 assay (a) and flow cytometry analysis (b) of LO2 cells before and after the incubation with the three BP nanosheets. (a) Timedependent viability (12 h, 24 h, 48 h) of LO2 cells after the incubation with L-BP, M-BP, and S-BP at different concentrations (6.3, 12.5, 25.0 and $50.0 \mu \mathrm{g} \mathrm{mL}^{-1}$ ). The data are represented as means \pm standard deviation. (b) Flow cytometry analysis of LO2 cells before and after the incubation with $25.0 \mu \mathrm{g} \mathrm{mL} \mathrm{m}^{-1}$ of L-BP, M-BP, and S-BP for $24 \mathrm{~h}$. The changes in cell apoptosis and necrosis are negligible. 


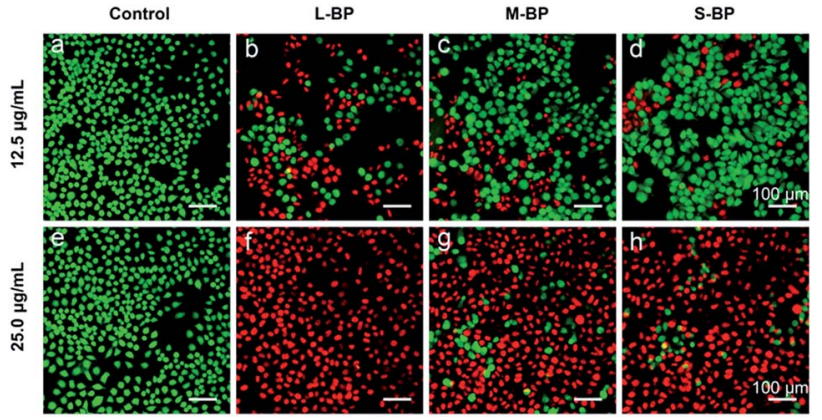

Fig. 5 Comparison of the photothermal ablation of MCF-7 cells after the addition of L-BP, M-BP, S-BP (12.5 and $\left.25.0 \mu \mathrm{g} \mathrm{mL}^{-1}\right)$ and illumination with an NIR laser $\left(808 \mathrm{~nm}, 1.0 \mathrm{~W} \mathrm{~cm}^{-2}\right)$ for $15 \mathrm{~min}$. Fluorescence images of cells stained with calcein AM (live cells, green fluorescence) and PI (dead cells, red fluorescence).

(808 $\mathrm{nm}, 1.0 \mathrm{~W} \mathrm{~cm}^{-2}$ ) for $15 \mathrm{~min}$. Afterwards, the cells are determined by the Live/Dead staining assay, where the calcein $\mathrm{AM} / \mathrm{PI}$ co-staining can differentiate the live/dead cells by green/ red fluorescence. It is clear from Fig. 5 that the photothermal therapy effects of BP nanosheets to MCF-7 cells are obvious. All the three kinds of BP nanosheets can ablate most of the MCF-7 cells at a concentration of $25.0 \mu \mathrm{g} \mathrm{mL}^{-1}$. More interestingly, the effects of BP nanosheets are size-dependent. When the concentration of various BP nanosheets is $12.5 \mu \mathrm{g} \mathrm{mL}^{-1}, \mathrm{~L}-\mathrm{BP}$ is much more effective than M-BP and S-BP for photothermal ablation of MCF-7 cells. This result is consistent with the previous results illustrated in Fig. $2 \mathrm{a}-\mathrm{d}$ that L-BP has highest photothermal performance.

\section{Conclusion}

In this study, we have successfully prepared three kinds of BP nanosheets with different sizes by using an ameliorated liquid exfoliation technique. The various BP nanosheets as-prepared are characterized for their physical/chemical properties, then examined for their effects to human normal hepatocyte. The results from optical observation, Live/Dead staining assay, CCK8 assay and flow cytometry analysis all demonstrate that the various BP samples are excellent in cytocompatibility. As BP is a promising candidate for photothermal therapy, all the three kinds of BP nanosheets are further investigated for their photothermal effects for cancer cells ablation. Interestingly, it is found that the photothermal therapy effects of BP nanosheets are size-dependent. On account of the high efficiency for photothermal conversion, L-BP is much more effective than M-BP and S-BP for the photothermal cancer therapy.

\section{Acknowledgements}

The authors gratefully acknowledge financial support from National Natural Science Foundation of China (NSFC) No. 31470044, 81371169 and 51672305, Frontier Research Key Project of the Chinese Academy of Sciences No. QYZDB-SSWSLH034 and Hong Kong Research Grants Council (RGC) General Research Funds (GRF) No. CityU 11301215.

\section{Notes and references}

1 P. W. Bridgman, J. Am. Chem. Soc., 1914, 36, 1344-1363.

2 D. M. Warschauer, J. Appl. Phys., 1963, 34, 1853-1860.

3 H. Liu, A. T. Neal, Z. Zhu, Z. Luo, X. F. Xu, D. Tomanek and P. D. Ye, ACS Nano, 2014, 8, 4033-4041.

4 F. N. Xia, H. Wang and Y. C. Jia, Nat. Commun., 2014, 5, 4458.

5 J. Kang, J. D. Wood, S. A. Wells, J. H. Lee, X. L. Liu, K. S. Chen and M. C. Hersam, ACS Nano, 2015, 9, 3596-3604.

6 L. K. Li, Y. J. Yu, G. J. Ye, Q. Q. Ge, X. D. Ou, H. Wu, D. L. Feng, X. H. Chen and Y. B. Zhang, Nat. Nanotechnol., 2014, 9, 372-377.

7 H. Liu, A. T. Neal, Z. Zhu, Z. Luo, X. Xu, D. Tomanek and P. D. Ye, ACS Nano, 2014, 8, 4033-4041.

8 Z. Sun, H. Xie, S. Tang, X. F. Yu, Z. Guo, J. Shao, H. Zhang, H. Huang, H. Wang and P. K. Chu, Angew. Chem., 2015, 54, 11526-11530.

9 D. J. Late, ACS Appl. Mater. Interfaces, 2015, 7, 5857-5862.

10 D. J. Late, Microporous Mesoporous Mater., 2016, 225, 494503.

11 A. S. Pawbake, M. B. Erande, S. R. Jadkar and D. J. Late, $R S C$ Adv., 2016, 6, 76551-76555.

12 M. Buscema, D. J. Groenendijk, S. I. Blanter, G. A. Steele, H. S. J. van der Zant and A. Castellanos-Gomez, Nano Lett., 2014, 14, 3347-3352.

13 S. Das, W. Zhang, M. Demarteau, A. Hoffmann, M. Dubey and A. Roelofs, Nano Lett., 2014, 14, 5733-5739.

14 L. Wang, Z. Sofer and M. Pumera, ChemElectroChem, 2015, 2, 324-327.

15 J. S. Qiao, X. H. Kong, Z. X. Hu, F. Yang and W. Ji, Nat. Commun., 2014, 5, 4475.

16 M. B. Erande, S. R. Suryawanshi, M. A. More and D. J. Late, Eur. J. Inorg. Chem., 2015, 19, 3102-3107.

17 S. R. Suryawanshi, M. A. More and D. J. Late, $R S C A d v ., 2016$, 6, 112103-112108.

18 S. R. Suryawanshi, M. A. More and D. J. Late, J. Vac. Sci. Technol., B: Nanotechnol. Microelectron.: Mater., Process., Meas., Phenom., 2016, 34, 041803.

19 S. Zhang, J. Yang, R. J. Xu, F. Wang, W. F. Li, M. Ghufran, Y. W. Zhang, Z. F. Yu, G. Zhang, Q. H. Qin and Y. R. Lu, ACS Nano, 2014, 8, 9590-9596.

20 J. Zhang, H. J. Liu, L. Cheng, J. Wei, J. H. Liang, D. D. Fan, J. Shi, X. F. Tang and Q. J. Zhang, Sci. Rep., 2014, 4, 6452.

21 J. Na, Y. T. Lee, J. A. Lim, D. K. Hwang, G. T. Kim, W. K. Choi and Y. W. Song, ACS Nano, 2014, 8, 11753-11762.

22 Y. C. Du, H. Liu, Y. X. Deng and P. D. Ye, ACS Nano, 2014, 8, 10035-10042.

23 C. Z. Zhang, N. Mahmood, H. Yin, F. Liu and Y. L. Hou, Adv. Mater., 2013, 25, 4932-4937.

24 C. M. Park and H. J. Sohn, Adv. Mater., 2007, 19, 2465-2468. 25 W. N. Zhu, M. N. Yogeesh, S. X. Yang, S. H. Aldave, J. S. Kim, S. Sonde, L. Tao, N. S. Lu and D. Akinwande, Nano Lett., 2015, 15, 1883-1890.

26 D. Xiang, C. Han, J. Wu, S. Zhong, Y. Y. Liu, J. D. Lin, X. A. Zhang, W. P. Hu, B. Ozyilmaz, A. H. C. Neto, A. T. S. Wee and W. Chen, Nat. Commun., 2015, 6, 6485. 
27 Y. Saito and Y. Iwasa, ACS Nano, 2015, 9, 3192-3198.

28 Y. Yang, J. Gao, Z. Zhang, S. Xiao, H. H. Xie, Z. B. Sun, J. H. Wang, C. H. Zhou, Y. W. Wang, X. Y. Guo, P. K. Chu and X. F. Yu, Adv. Mater., 2016, 28, 8937-8944.

29 J. Dai and X. C. Zeng, J. Phys. Chem. Lett., 2014, 5, 1289-1293. 30 S. H. Lin, S. H. Liu, Z. B. Yang, Y. Y. Li, T. W. Ng, Z. Q. Xu, Q. L. Bao, J. H. Hao, C. S. Lee, C. Surya, F. Yan and S. P. Lau, Adv. Funct. Mater., 2016, 26, 864-871.

31 M. Engel, M. Steiner and P. Avouris, Nano Lett., 2014, 14, 6414-6417.

32 M. Q. Huang, M. L. Wang, C. Chen, Z. W. Ma, X. F. Li, J. B. Han and Y. Q. Wu, Adv. Mater., 2016, 28, 3481-3485.

33 M. B. Erande, M. S. Pawar and D. J. Late, ACS Appl. Mater. Interfaces, 2016, 8, 11548-11556.

34 C. X. Hao, B. C. Yang, F. S. Wen, J. Y. Xiang, L. Li, W. H. Wang, Z. M. Zeng, B. Xu, Z. S. Zhao, Z. Y. Liu and Y. J. Tian, Adv. Mater., 2016, 28, 3194-3201.

35 H. Wang, X. Z. Yang, W. Shao, S. C. Chen, J. F. Xie, X. D. Zhang, J. Wang and Y. Xie, J. Am. Chem. Soc., 2015, 137, 11376-11382.

36 J. Shao, H. Xie, H. Huang, Z. Li, Z. Sun, Y. Xu, Q. Xiao, X. F. Yu, Y. Zhao, H. Zhang, H. Wang and P. K. Chu, Nat. Commun., 2016, 7, 12967.

37 C. Sun, L. Wen, J. Zeng, Y. Wang, Q. Sun, L. Deng, C. Zhao and Z. Li, Biomaterials, 2016, 91, 81-89.

38 J. D. Wood, S. A. Wells, D. Jariwala, K. S. Chen, E. Cho, V. K. Sangwan, X. L. Liu, L. J. Lauhon, T. J. Marks and M. C. Hersam, Nano Lett., 2014, 14, 6964-6970.
39 Y. T. Zhao, H. Y. Wang, H. Huang, Q. L. Xiao, Y. H. Xu, Z. N. Guo, H. H. Xie, J. D. Shao, Z. B. Sun, W. J. Han, X. F. Yu, P. H. Li and P. K. Chu, Angew. Chem., Int. Ed., 2016, 55, 5003-5007.

40 J. Dai and X. C. Zeng, RSC Adv., 2014, 4, 48017-48021.

41 H. Yue, W. Wei, Z. Yue, B. Wang, N. Luo, Y. Gao, D. Ma, G. Ma and Z. Su, Biomaterials, 2012, 33, 4013-4021.

42 Z. Guo, H. Zhang, S. Lu, Z. Wang, S. Tang, J. Shao, Z. Sun, H. Xie, H. Wang, X.-F. Yu and P. K. Chu, Adv. Funct. Mater., 2015, 25, 6996-7002.

43 J. T. Robinson, S. M. Tabakman, Y. Liang, H. Wang, H. S. Casalongue, D. Vinh and H. Dai, J. Am. Chem. Soc., 2011, 133, 6825-6831.

44 L. Cheng, J. Liu, X. Gu, H. Gong, X. Shi, T. Liu, C. Wang, X. Wang, G. Liu, H. Xing, W. Bu, B. Sun and Z. Liu, Adv. Mater., 2014, 26, 1886-1893.

45 D. Gopalakrishnan, D. Damien and M. M. Shaijumon, ACS Nano, 2014, 8, 5297-5303.

46 V. Stone, H. Johnston and R. P. Schins, Crit. Rev. Toxicol., 2009, 39, 613-626.

47 Y. Zhang, S. F. Ali, E. Dervishi, Y. Xu, Z. Li, D. Casciano and A. S. Biris, ACS Nano, 2010, 4, 3181-3186.

48 W. H. De Jong, W. I. Hagens, P. Krystek, M. C. Burger, A. J. Sips and R. E. Geertsma, Biomaterials, 2008, 29, 19121919.

49 J. H. Liu, S. T. Yang, H. F. Wang, Y. L. Chang, A. N. Cao and Y. F. Liu, Nanomedicine, 2012, 7, 1801-1812.

50 S. M. Hussain, K. L. Hess, J. M. Gearhart, K. T. Geiss and J. J. Schlager, Toxicol. In Vitro, 2005, 19, 975-983. 